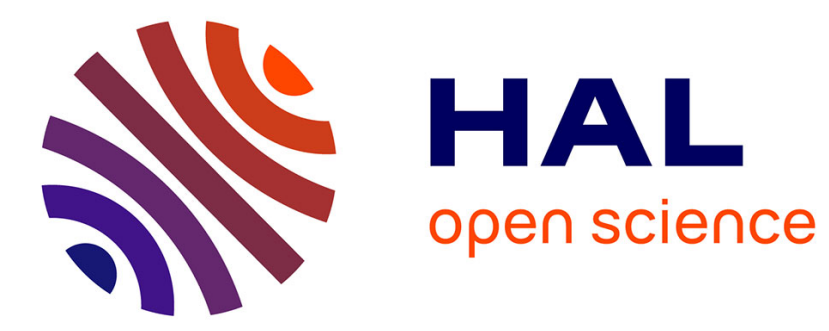

\title{
Experimental validation of an eddy current probe dedicated to the multi-frequency imaging of bore holes
}

Pierre-Yves Joubert, Eric Vourc'H, Vincent Thomas

\section{To cite this version:}

Pierre-Yves Joubert, Eric Vourc'H, Vincent Thomas. Experimental validation of an eddy current probe dedicated to the multi-frequency imaging of bore holes. Sensors and Actuators A: Physical , 2012, 185, pp.132-138. 10.1016/j.sna.2012.07.009 . hal-00829024

\section{HAL Id: hal-00829024 https://hal.science/hal-00829024}

Submitted on 1 Jun 2013

HAL is a multi-disciplinary open access archive for the deposit and dissemination of scientific research documents, whether they are published or not. The documents may come from teaching and research institutions in France or abroad, or from public or private research centers.
L'archive ouverte pluridisciplinaire HAL, est destinée au dépôt et à la diffusion de documents scientifiques de niveau recherche, publiés ou non, émanant des établissements d'enseignement et de recherche français ou étrangers, des laboratoires publics ou privés. 


\title{
EXPERIMENTAL VALIDATION OF AN EDDY CURRENT PROBE DEDICATED TO THE MULTI-FREQUENCY IMAGING OF BORE HOLES
}

\author{
P.-Y. JOUBERT ${ }^{1}$, E. VOURC'H ${ }^{1}$, V. THOMAS ${ }^{1}$ \\ ${ }^{1}$ IEF, CNRS UMR 8622, Univ Paris Sud-P11, Bât 220, F-91405 Orsay Cedex, France \\ ${ }^{2}$ SATIE, ENS Cachan, CNRS, UniverSud, 61 Av. du Président Wilson, 94235 CACHAN Cedex, France
}

\section{Article info :}

Submitted on February 2012

Published on October 2012

\section{Key words :}

Eddy currents, non destructive evaluation, imaging system, bore holes, sensor array, defect detection, defect characterization.

Authors E-mail addresses :

pierre-yves.joubert@u-psud.fr

(P.-Y. Joubert)

eric.vourch@satie.ens-cachan.fr

(E. Vourc'h)

\begin{abstract}
:
This paper deals with the experimental characterization of an array probe dedicated to the eddy current imaging of sub-millimetric surface breaking defects appearing in bore holes of metallic parts. The probe is constituted of a large inducer generating an uniformly oriented EC flow within the inspected material, and a sensing array probe featuring bobbin coils to sense the radial component of the magnetic field resulting from the eddy currents / defects interactions within the wall of the bore hole. The probe was designed with accurate defect characterization in view, i.e. to provide multi-frequency and high spatial resolution images with a reduced acquisition time, so as to enhance the informative content of the acquired eddy current data. An experimental set-up was build in order to validate the imaging performances of such a probe. A prototype featuring a large inducer and a single sensing coil which can be accurately positioned in the sensing area, has been developed in order to evaluate the sensing performances as well as to study the influence of the sensing array configuration on the imaging performances. The experimental results demonstrate a good sensing ability of the designed probe in the 10 $\mathrm{kHz}-800 \mathrm{kHz}$ frequency range, with peak-signal-to-noise ratios higher than $36 \mathrm{~dB}$ at 10 $\mathrm{kHz}$ (and $62 \mathrm{~dB}$ at $800 \mathrm{kHz}$ ) for defects featuring dimensions as small as $0.4 \times 0.2 \times 0.2$ $\mathrm{mm}^{3}$. Furthermore, a staggered row arrangement of the sensing array was proposed so as to significantly reduce the error due to the sampling step resulting from the pickup coils geometry (from $35 \%$ to less than $9 \%$ in the worst case). The experimental evaluation of the probe provides promising prospects for the accurate characterization of defects, by means of advanced multifrequency signal processing algorithms.
\end{abstract}

\section{Introduction}

The demand for faster non destructive evaluations (NDE) of conductive structures and for the accurate characterization of the defects that may be detected is a motivation toward the development of eddy current (EC) imaging systems. These systems aim at providing an automated, quantitative and accurate diagnosis of the inspected parts, starting from the collected EC data. To do so, they require that i) a dedicated probe provides accurate and relevant EC data, ii) accurate and computationally efficient modeling algorithms are derived so as to model the physical interactions between the part and the EC probe iii) the inverse problem can be solved, i.e. the quantitative estimation of the defect within the inspected part is carried out, starting from the collected EC data and using the modeling algorithms. However, inverse problems in eddy current NDE are known to be "ill posed" $[1,2]$, meaning that the solution may not be unique or may even not exist. This problem is made worse by the incompleteness of the EC data that are available [3] when conventional EC probes are used. In order to overcome this major drawback, the first step consists in providing more relevant EC data. This is why efforts have been made in the last decade to design EC probes which provide enhanced EC data. Among them, probes using large bandwidth excitation systems (pulsed eddy current systems [4]) rather than a harmonic excitation have been proposed. Also, probes implementing rotating excitation fields have been designed so as to investigate the inspected part under various "viewpoints" [5,6]. Furthermore, recent progresses made in the miniaturization of the magnetic sensors and in the use of integrated technologies enable advanced array probes to be built [5-11]. Such array probes are suitable for the design of EC imaging systems since they allow two dimensional C-scan images to be accurately collected, with an acquisition time reduced if compared to the $\mathrm{EC}$ data provided by single sensor probes which require more fastidious mechanical scanning [8]. A variety of such EC array probes has been reported. These probes implement various magnetic field sensors such as Hall effect sensors [7], coils [8,9] or high sensitivity sensors such as giant magneto resistances (GMR) $[10,11]$. Moreover, depending on the size of the sensors and also, as far as coils are concerned, on the possibility of using a flexible support, probes matching complex geometries can be designed [9]. Finally, high resolution C-scans also have been provided by magneto-optic EC system $[12,13]$ dedicated to the imaging of plane parts. In these different devices, the EC 
flowing within the inspected part can either be induced locally, using elements of the array probe as local transmitters $[8,9]$, or induced "globally", i.e. within the whole inspection area, using a large EC inducer [11]. The latter can be designed taking advantage of the symmetries of the inspected part in order i) to optimize the electromagnetic coupling with the part, ii) to reduce perturbation parameters such as the geometry changes in the part, iii) and/or to reduce the direct coupling between the inducer and the magnetic field sensor array [13]. Thanks to the use of such global inducers, imaging devices that are only sensitive to the magnetic field components that are due to $\mathrm{EC} /$ defects interactions may be designed $[11,13]$. For example, such a configuration proposed in [14] or [15] enables to induce a uniform eddy current flow in the inspected area, so that the measurement system is self-nulling (measured EC data are non-zero only in the presence of a defect). Recently, the authors have applied this approach to design an EC array probe dedicated to the imaging of bore holes featuring sub-millimetric surface-breaking defects. The configuration of the proposed probe aims at allowing efficient acquisition of relevant data to be carried out (thanks to a sensing array), as well as computationally efficient defect reconstruction algorithms to be implemented. The design of the probe itself, including the study of the influence of the sensing element technology, was carried out by the means finite element computations and presented in [16]. In paper [17], the authors have proposed an inversion algorithm, developed in a total variation regularized optimization framework, able to carry out the three-dimensional reconstruction of surface breaking defects in the bore-hole, starting from EC data simulated in a large frequency bandwidth. In this paper, we report on the experimental validation and characterization of such an EC array probe dedicated to bore-hole imaging, with multi-frequency reconstruction of defects in view. The paper is organized as follows. Section 2 provides the probe features and the description of the experimental setup that is implemented. The characterization of the probe is reported in section 3: experimental magnetic flux density cartographies (C-scans) are provided and compared to simulated data. Moreover, the multi-frequency operation of the probe is characterized. Then, the influence of the structure of the array probe is studied with regards to the sampling of the collected C-scan. Finally, concluding remarks are given in section 4 .

\section{EC imaging array probe and experimental set-up}

\subsection{Structure and basic principle of the probe}

The proposed EC probe aims at providing rapidly and accurately sampled C-scan images relative to surface breaking defects. Given the cylindrical geometry of the part to be imaged, this probe was designed based on a global inducer consisting in a large coil coaxial to the bore hole (Figure 1) which allows a good electromagnetic coupling with the part to be achieved. Thanks to this configuration, a uniformly oriented EC flow is induced within the inner wall the bore hole along the $\vec{\theta}$ axis (Figure 1.B). In the absence of a defect, for symmetry reasons, no radial component of the magnetic field is generated. Conversely, the presence of a surface

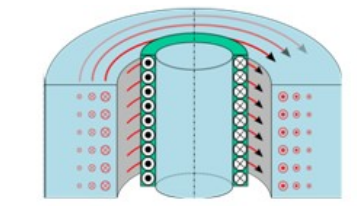

A. Global inducer and $\mathrm{EC}$ flow

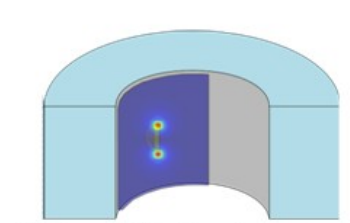

C. Magnetic field cartography

Generated at the vicinity of the defect

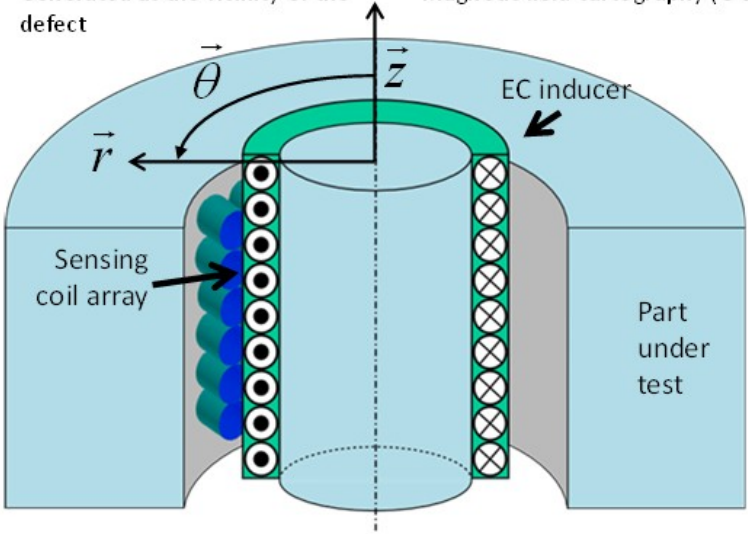

E. Structure of the EC imaging probe.

Figure 1. Structure and basic principle of the EC imaging probe.

breaking defect will locally modify the EC flow (Figure 1.B). Consequently, new magnetic field components will appear in the vicinity of the defect and, among them, a non-zero radial component will arise (Figure 1.C) along the $\vec{r}$ axis. Sensing this particular component seems therefore particularly judicious since it is null in the absence of a defect. Indeed, in this way, there is no direct coupling between the inducer and the magnetic field sensor.

This configuration enhances the sensitivity of the probe to the defects [16], and enables the use of high gain amplifiers to provide low noise measurement data. In order to rapidly sample the two-dimensional distribution (C-scan) of the radial magnetic field, the use of a sensing coil array is considered (Figure 1.D). The resulting configuration of the whole EC probe is as depicted in Figure 1.E.

\subsection{Probe features}

A prototype of the sensor array probe was build for experimental validation purposes. The inducer of the probe is constituted of $58 \mathrm{~mm}$ long and $48.8 \mathrm{~mm}$ diameter single layered bobbin-coil featuring 76 turns wound with a $1.25 \mathrm{~mm}$ diameter copper wire, so as to reduce the capacitance of the inducer [18]. This inducer was characterized by means of an HP4192A impedance analyzer. The resistance of the inducer, measured at $10 \mathrm{~Hz}$ was found to be $R_{\text {inducer }}=212 \Omega \pm 2 \mathrm{~m} \Omega$, the self inductance $L_{\text {inducer }}=44.22 \mu \mathrm{H} \pm 0.01 \mathrm{~Hz}$ (at $100 \mathrm{kHz}$ ), and the stray capacitance, measured at $10 \mathrm{MHz}$ was found to 


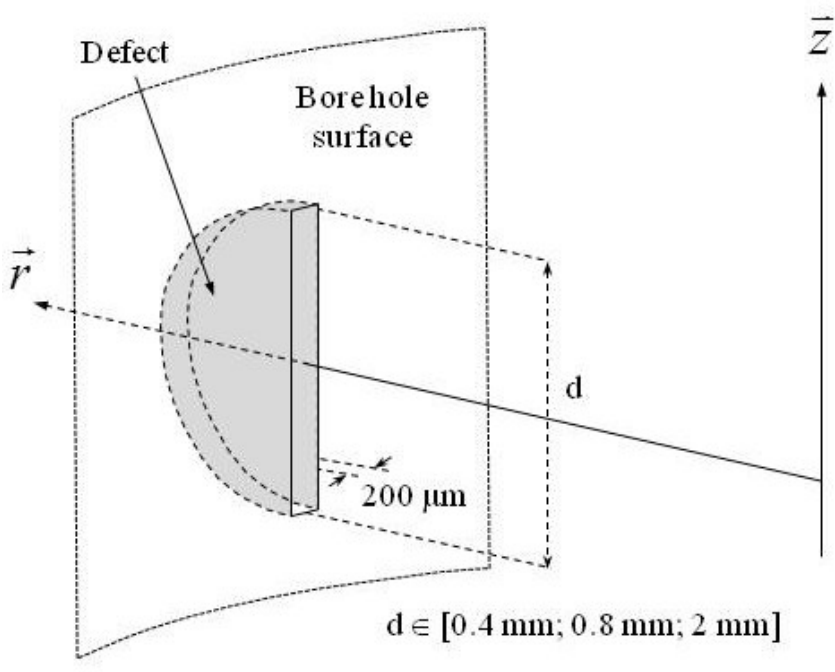

Figure 2. Surface breaking defect.

be $C_{\text {inducer }}=28.8 \mathrm{pF} \pm 0.2 \mathrm{pF}$. In practice, these features enable the inducer to correctly operate from a few $\mathrm{kHz}$ up to 800 $\mathrm{kHz}$, and large frequency bandwidth operations may be envisaged. The elements of the sensing array are pickup coils of $1.4 \mathrm{~mm}$ long, of $1.4 \mathrm{~mm}$ outer diameter, and featuring 460 turns distributed on 10 layers of 46 turns, wound with a $50 \mu \mathrm{m}$ diameter copper wire. This kind of mini-bobbin coils features a bandwidth larger than $10 \mathrm{MHz}$ and a sensitivity higher than $350 \mathrm{~V} / \mathrm{T}$ at $100 \mathrm{kHz}$. Since these features are sufficient for the sensing of surface breaking defects, mini-coils have been chosen as pick-up elements in this study rather than solid state sensors such as Hall-effect sensors [19] or giant effect magneto-resistances. Indeed, these solid state sensors are sensitive to in-plane magnetic field components, and may be found more difficult to integrate in a pickup array than bobbin coils, considering that it is the radial component of the magnetic field in the bore hole that is to be imaged.

\subsection{Experimental set-up}

The part under test is a bore hole of diameter $52 \mathrm{~mm}$ machined in a $170 \mathrm{~mm} \times 100 \mathrm{~mm} \times 50 \mathrm{~mm}$ laboratory made mock-up, made of a 2024 T3 aluminum alloy, representative of an aeronautic part, which features an electrical conductivity of 17.6 MS/m [20]. The part features three defects denoted $D_{l}$, $D_{2}$ and $D_{3}$ machined using an electrical discharged machining (EDM) process. The defects feature a semi-circular shape, a $200 \mu \mathrm{m}$ aperture and a diameter $d$ equal to $0.4 \mathrm{~mm}, 0.8 \mathrm{~mm}$ and $2 \mathrm{~mm}$ respectively (Figure 2 ). The defects are located in the middle of the cylindrical hole (far from the mouth of the bore hole) and their main orientation is along the $\vec{z}$ axis (Figure 2). The global inducer of the probe is introduced inside the bore hole (Figure 3) and fed with alternated currents of adjustable frequency through a power amplifier. A single pickup coil, oriented along the $\vec{r}$ axis, is fixed to a moving support and placed in the air-gap separating the inducer from the wall of the bore hole. Due to the configuration of the probe, to the dimensions of the constitutive parts, and to the positioning accuracy of the motorized axes of the robot used

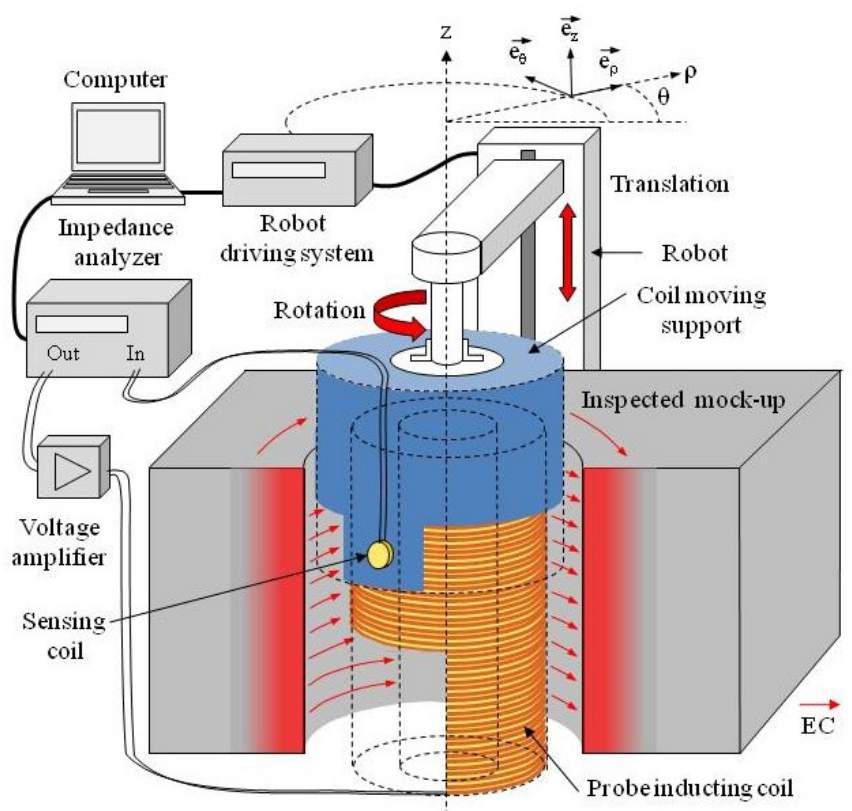

Figure 3. Experimental set-up.

to move the probe, the lift-off between the sensing coil and the wall of the bore hole was estimated to be $200 \mu \mathrm{m} \pm 20 \mu \mathrm{m}$. The pickup coil senses the radial component of the magnetic field and is moved along the $\vec{z}$ and $\vec{\theta}$ axes by means of a translation and a rotation PC controlled actuators, so as to build the CScan EC cartographies (Figure 3). The EC data are actually collected at the ends of the pickup coil using a PC controlled HP4192A impedance analyzer by means of lock-in amplification.

This single sensing pickup coil configuration was preferred to the use of an actual sensor array for the sake of simplicity in the implementation of this proof of concept set-up. The position of the pickup coil is accurately adjustable along the $\vec{Z}$ and $\vec{\theta}$ axes, using the PC controlled actuators, so that various array sensor configurations may be simulated and studied. Indeed, the moving of the pickup coil enables the acquisition of the EC C-scan within the defect area, denoted $I$, with adjustable scanning steps of $s_{z}=m . d z$ and $s_{\theta}=n \cdot R . d \theta$, with $m, n \in\{1,2,3, \ldots \mathrm{N}\}$, and where $d z=12.5 \mu \mathrm{m}$ and R. $d \theta=50 \mu \mathrm{m}$.

\section{Implementation and characterization of the EC imaging probe}

\subsection{First experimental results}

The implementation of the EC imaging probe was firstly carried out at $90 \mathrm{kHz}$ considering the defects $D_{1}, D_{2}$, and $D_{3}$. The resulting $\mathrm{C}$-scan images $I$ are complex and constituted of in-phase and in-quadrature images, respectively denoted $I_{p}$ and $I_{q}$, obtained by reference to the phase-shift of the exciting current, so that $I=I_{p}+j \cdot I_{q}$. They are presented in Figure 4, Figure 5, and Figure 6 for defects $D_{1}, D_{2}$, and $D_{3}$ respectively. These images are obtained in a $4.4 \times 4.4 \mathrm{~mm}^{2}$ area, with scanning steps featured by $m=8$ and $n=2$ (i.e. $s_{z}=s_{\theta}=0.1$ 
Experimental C-scan for $\mathrm{D}_{1}$

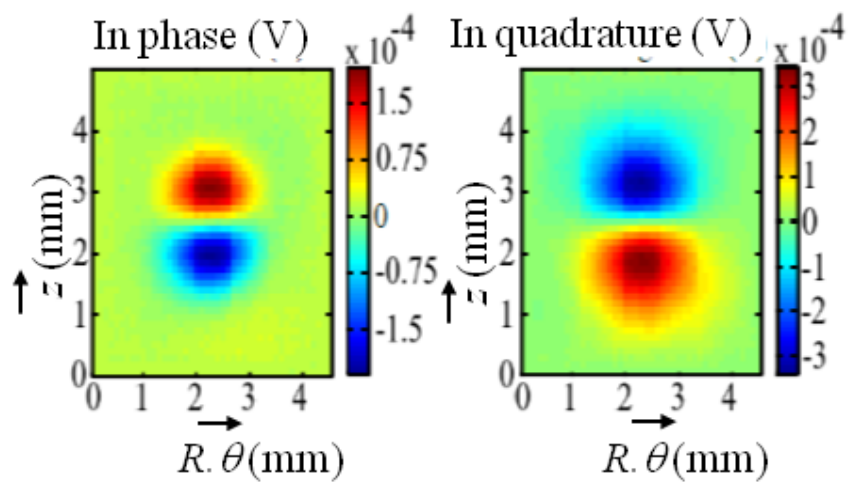

Computed C-scan for $\mathrm{D}_{1}$

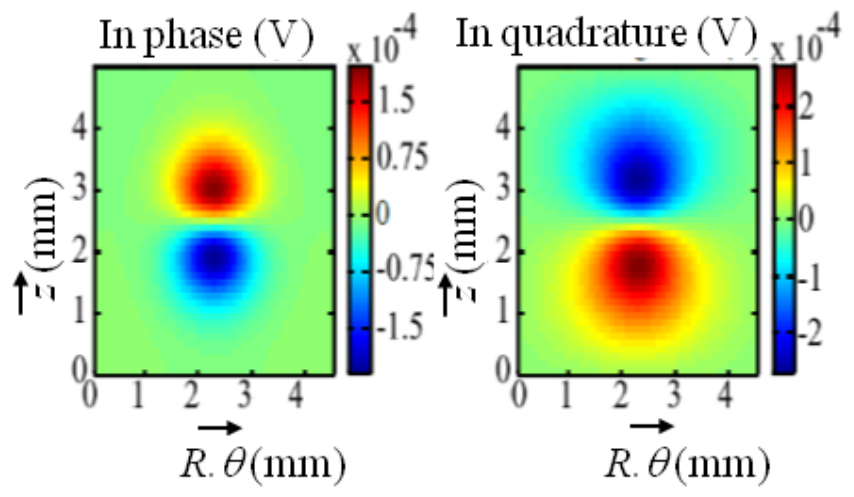

Figure 4. EC C-scans for defect $D_{l}$, at $90 \mathrm{kHz}$.

Experimental C-scan for $\mathrm{D}_{2}$

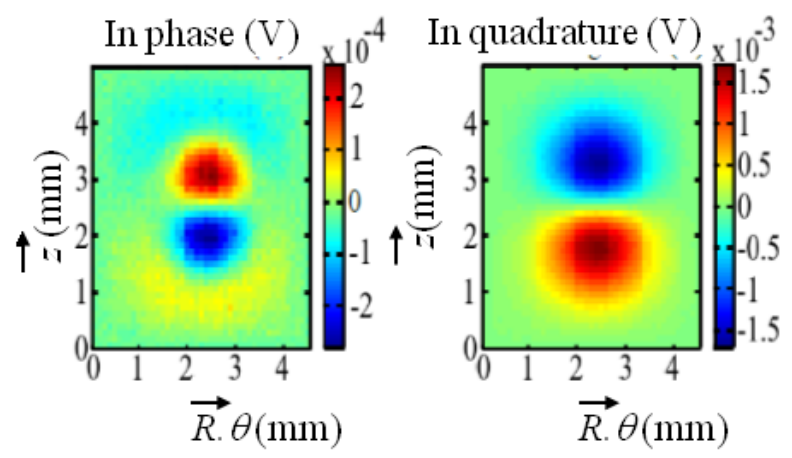

Computed C-scan for $\mathrm{D}_{2}$

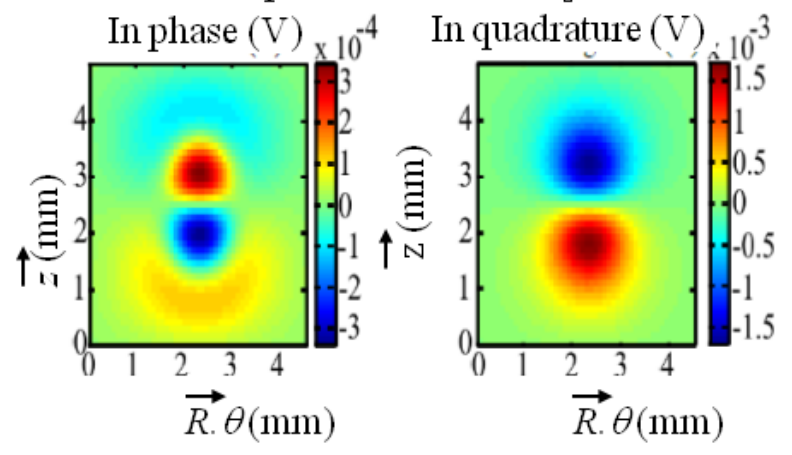

Figure 5. EC C-scans for defect $D_{2}$, at $90 \mathrm{kHz}$.

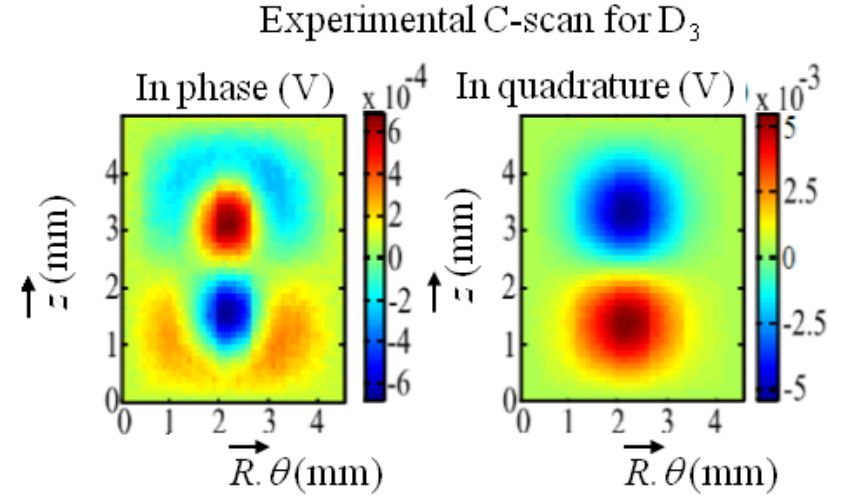

Computed C-scan for $\mathrm{D}_{3}$

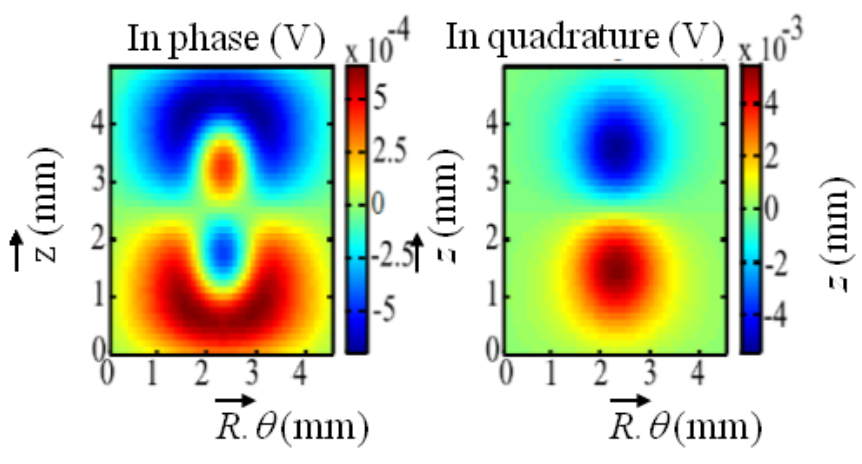

Figure 6. EC C-scans for defect $D_{3}$, at $90 \mathrm{kHz}$.

$\mathrm{mm})$. For the sake of validation, the obtained C-scans where compared to simulated data, obtained by means of finite element computations of the same configuration in which the lift-off of the sensing coil is assumed to be $200 \mu \mathrm{m}$. Lissajous plots relative to a cut line passing above the defects along the $\vec{z}$ axis are also provided in Figure 7. In both representations, one can note a fair agreement between experimental and computed data whatever the considered defect. The small amplitude or phase difference that can be noticed may originate from a mis-positioning (variation of lift-off) of the sensing coil or, as far as the smallest defects is concerned, from the tolerance margin of the defects manufacturing. Nevertheless, these preliminary results validate the experimental feasibility of the EC imaging probe.

The imaging probe is designed to operate in a large frequency bandwidth, since previous works have shown that enhanced defect reconstruction results were obtained starting from multifrequency EC data [17]. Considering the penetration depth $\delta$ of the EC within the material, which may be approximated by [21]:

$$
\delta=\frac{1}{\sqrt{\sigma \mu \pi f}}
$$

and considering the larger defect to be imaged $\left(D_{3}, 1 \mathrm{~mm}\right.$ deep), one can conclude that frequencies ranging from less than $15 \mathrm{kHz}$ up to hundreds of $\mathrm{kHz}$ should be used to provide varied EC information relative to these surface-breaking defects. 

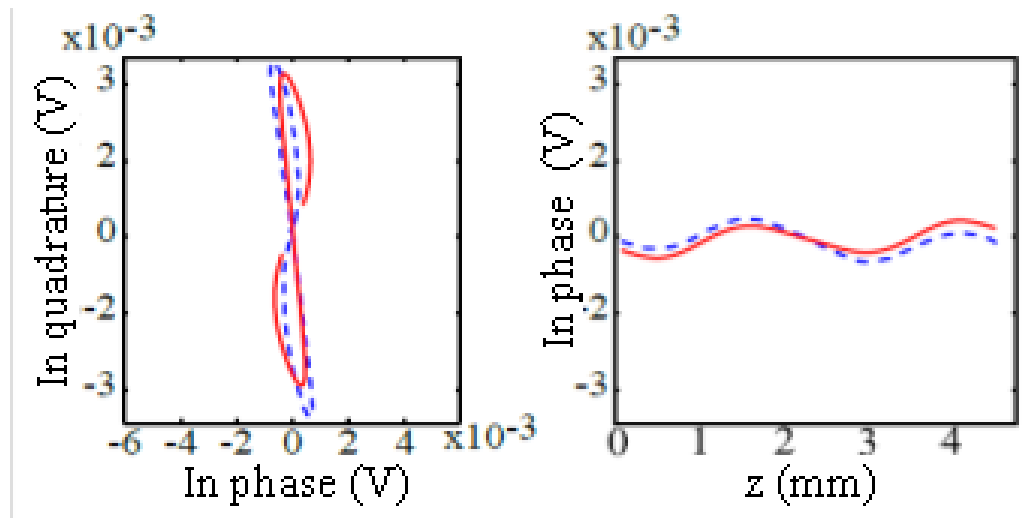

a)
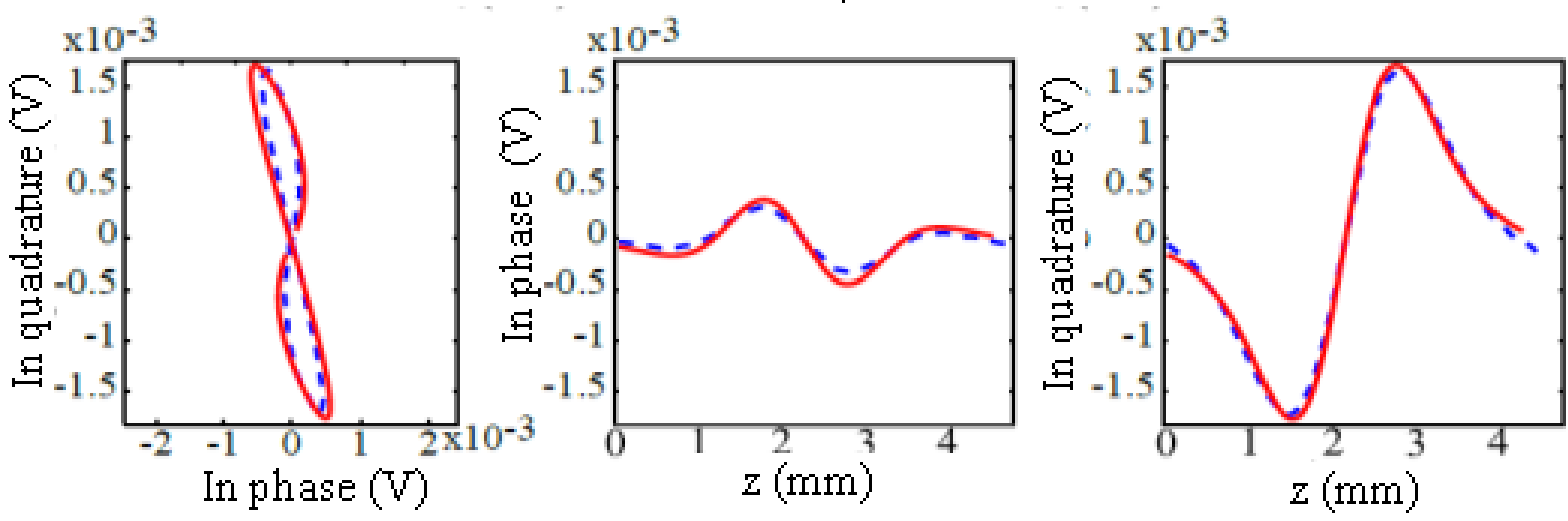

b)
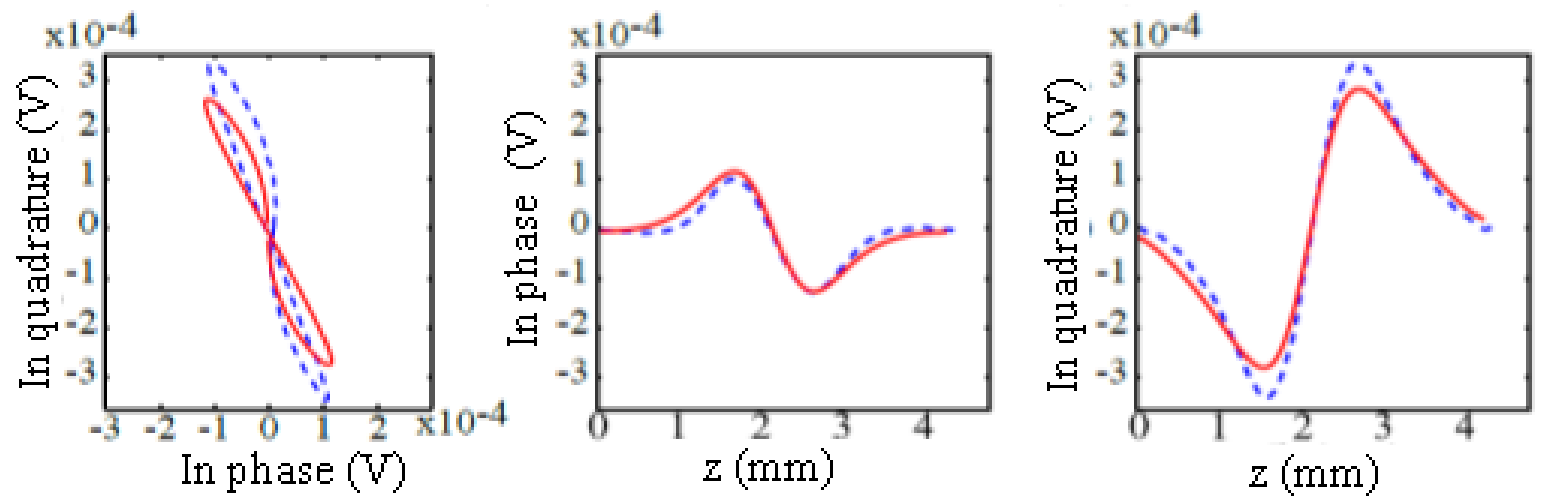

c)

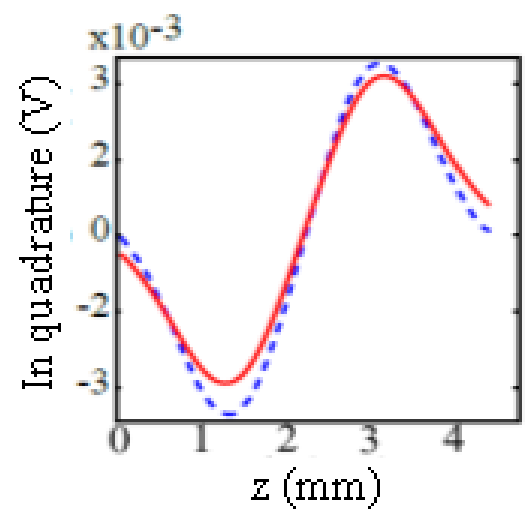

Figure 7. Lissajous plots, in-phase and in-quadrature signal obtained along a cut line parallel to the $\overrightarrow{\boldsymbol{z}}$ axis for a) defect $D_{3}$, b) defect $D_{2}$ and c) defect $D_{l}$, at $90 \mathrm{kHz}$. Solid lines are relative to simulations, dotted lines are relative to experiments.

In order to quantify the experimental sensing ability of the probe in that frequency bandwidth, we use the peak signal to noise ratio (PSNR), defined as [22]:

$$
\operatorname{PSNR}=20 \cdot \log \frac{\max (|I|)}{\sqrt{\sigma_{p}^{2}+\sigma_{q}^{2}}}
$$

where $\max (|I|)$ is the peak value of the modulus of $I$ taken above the defect signature, and $\sigma_{p}$ and $\sigma_{q}$ are the standard deviations of the noise, assumed to be Gaussian, which are estimated from 80 pixels taken in defect-free areas comprised in the $I_{p}$ and $I_{q}$ images, respectively. As an example, the estimation of the noise in the case of defect $D_{2}$ at $90 \mathrm{kHz}$ (Figure 4) leads to $\sigma_{p}=2.2 \mu \mathrm{V}$ and $\sigma_{q}=2.3 \mu \mathrm{V}$. With $\max (|I|)=1.82 \mathrm{mV}$, the PSNR calculated using Eq. 2 is found to be $55.1 \mathrm{~dB}$.

The PSNR was estimated for the C-scan images obtained experimentally at frequencies varying in the $10-800 \mathrm{kHz}$ 
range, when considering the defects $D_{1}, D_{2}$ and $D_{3}$. The results are gathered in Table 1. One can note that in the considered frequency range, all the defects are correctly sensed (the PSNR being always higher than $36 \mathrm{~dB}$ ), which gives good prospects for the solving of the inverse problem required for the accurate characterization of defects [17]. Furthermore, the evolution of the maximum value of the defect signatures as a function of the defect depth is plotted for 3 different frequencies in Figure 8. One can note that the obtained curves are monotonous, that is to say defects of different diameters may be discriminated considering the value of $\max (|I|)$. Moreover, the sensitivity of the probe (the slope of the different curves) changes with the frequency. As a result, multi-frequency EC data may be used simultaneously so as to enhance the robustness of the discrimination between defects of different diameter.

Table 1. Estimation of the PSNR obtained on experimental data provided by the probe for various defects and excitation frequencies.

\begin{tabular}{|llllllll|}
\hline Frequency $(\mathrm{kHz})$ & 10 & 40 & 55 & 90 & 150 & 350 & 800 \\
\hline PSNR for $D_{l}(\mathrm{~dB})$ & 36.2 & 40.7 & 44.9 & 45.2 & 44.8 & 50.8 & 62.4 \\
\hline PSNR for $D_{2}(\mathrm{~dB})$ & 46.1 & 50.3 & 54.5 & 55.1 & 55.7 & 50.3 & 61.6 \\
\hline PSNR for $D_{3}(\mathrm{~dB})$ & 48.2 & 51.9 & 56.5 & 57.3 & 58.1 & 58.1 & 63.6 \\
\hline
\end{tabular}

\subsection{Effect of the sampling step}

The EC images considered in the previous sections (see Figure 4 to Figure 6 ) were obtained by moving a single pickup coil over the defect zone with $0.1 \mathrm{~mm} \times 0.1 \mathrm{~mm}$ spatial sampling steps. If a fixed sensing array featuring $1.4 \mathrm{~mm}$ diameter pickup coils was used instead of a single moving pickup coil, the obtained images would be rather different, since the sampling step, resulting from the size of the coils, would be significantly larger. As a result, the quality of the obtained sensed cartography might be altered, so as the performances of the defect characterization. Therefore, the influence of the sampling step as well as the coil arrangement of the array have to be evaluated. To do so, let us denote $I_{m, n}$ the C-scan image obtained with the sampling steps $s_{z}$ and $s_{\theta}$ featured by $m$ and $n$, as defined in section 2.3. The C-scan images shown in Figure 4 to Figure 6 are obtained with samplings steps of $s_{z}=s_{\theta}=0.1 \mathrm{~mm}$. They are therefore denoted $I_{8,2}$ and constitute the reference images in this study. Using an actual array probe would lead to consider sampling steps of larger dimensions than $0.1 \mathrm{~mm}$. Indeed, if the sensing array is constituted of $1.4 \mathrm{~mm}$ diameter pickup coils placed side by side, the resulting $\mathrm{C}$-scan images would be obtained with sampling steps of $s_{z}=s_{\theta}=1.4 \mathrm{~mm}$. These features may alter the sensing of the defects and deteriorate the defect characterization performances of the imaging system. In order to reduce the degradation due to large sampling steps, EC images may be interpolated before being processed by an inversion algorithm.

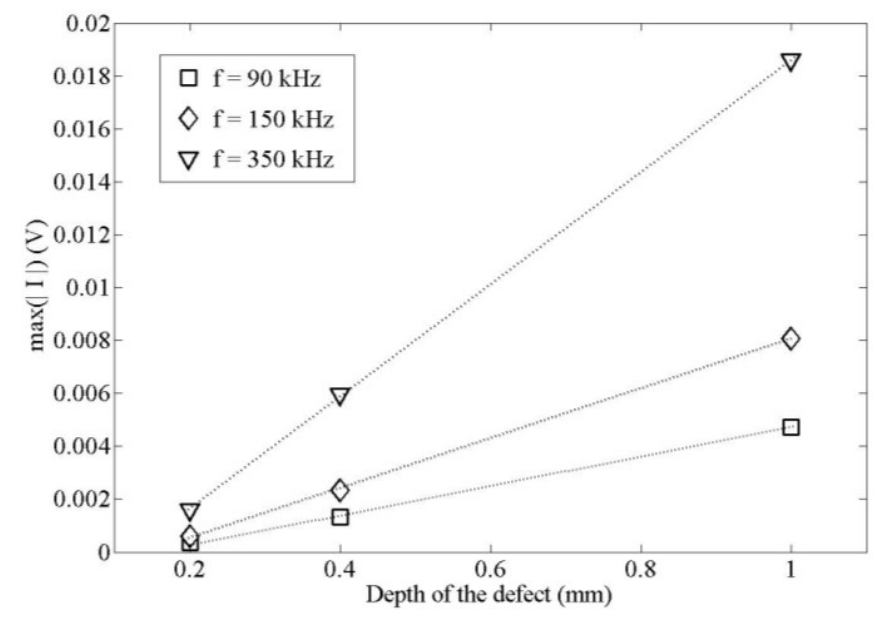

Figure 8. Evolution of the maximum value of $|I|$ with the depth of the defect, for different frequencies.

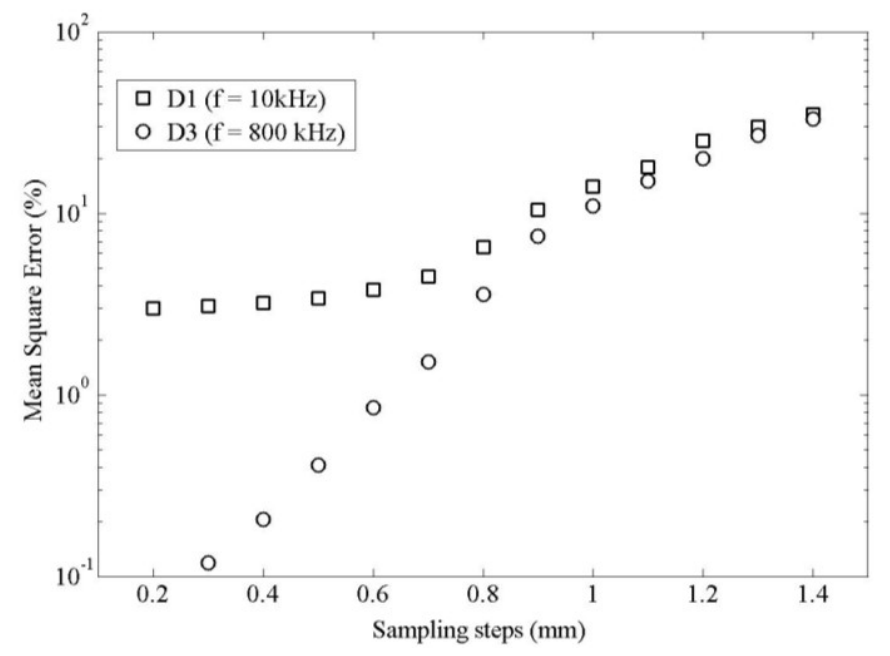

Figure 9. Mean square error versus sampling steps in two configurations: defect $D_{l}$ observed at $10 \mathrm{kHz}$ (worst case for detection) and defect $D_{3}$ observed at $800 \mathrm{kHz}$ (best case for detection).

In order to study the influence of the sampling effect onto the quality of the experimental EC images, we consider the interpolation error $\varepsilon_{m, n}$ defined as:

$$
\varepsilon_{m, n}=I_{8,2}-\widehat{I_{m, n}}
$$

where $\widehat{I_{m, n}}$ is the cubic interpolation of EC image $I_{m, n}$, carried out so as to match the sampling steps of the reference image $I_{8,2}$. Finally, the mean square error $\varepsilon_{\mathrm{MSE}}$ due to the sampling and interpolation, computed by comparison to the reference image, can be evaluated using:

$$
\varepsilon_{\mathrm{MSE}}=\frac{\sum_{\mathrm{k}, 1}\left|\varepsilon_{\mathrm{m}, \mathrm{n}}\right|^{2}}{\sum_{\mathrm{k}, \mathrm{l}}\left|I_{8,2}\right|^{2}}
$$

where $k$ and $l$ are the number of pixels of the EC image along the the $\vec{z}$ and $\vec{\theta}$ axes. 


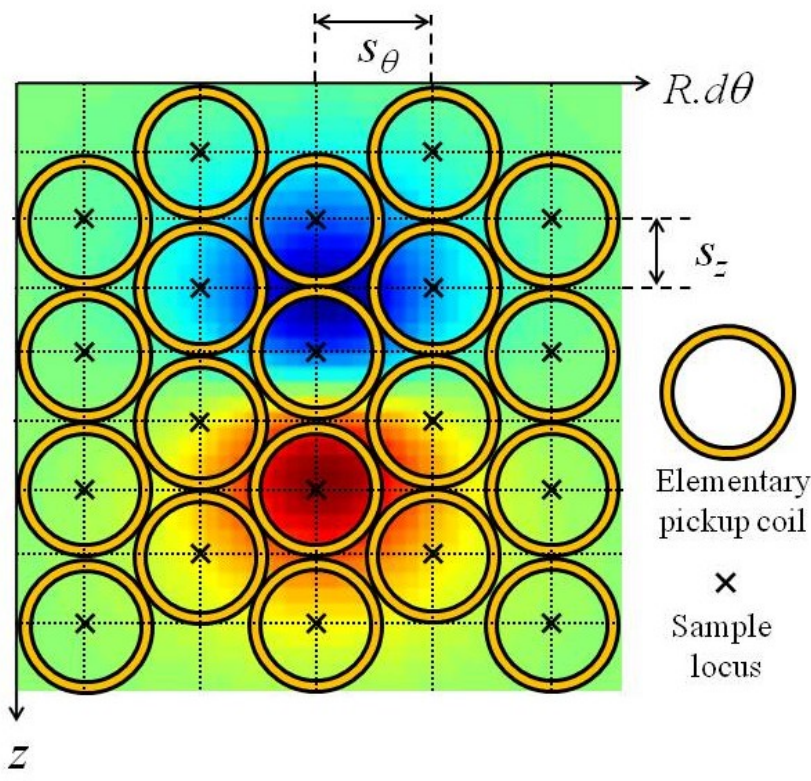

Figure 10. Staggered row arrangement of the pickup coils of the sensing array.

In this work, the influence of the sampling step was studied in two measurement configurations: the EC observation of the smallest defect $D_{l}$ at $10 \mathrm{kHz}$, which constitutes the worst case of detection, and the observation of defect $D_{3}$ at $800 \mathrm{kHz}$, which constitutes the best case of detection according to Table 1. In both configurations, the error was evaluated versus the sampling steps used to carry out the data acquisition. The used sampling steps range from $s_{z}=s_{\theta}=0.2 \mathrm{~mm}$ (i.e. $m=16$, $n=4)$ to $s_{z}=s_{\theta}=1.4 \mathrm{~mm}$ (i.e. $m=112, n=28$ ), the latter values being relative to the sampling steps provided by a sensing array constituted of 1.4 diameter sensing coils placed side by side. The obtained results are provided in Figure 9. In both configurations, one can observe that the error is increasing from less than a few percent up to $35 \%$ when the sampling steps are as large as $1.4 \mathrm{~mm}$. In order to keep the error lower than a few percent even in the worst defect configuration, one may use a staggered row arrangement of the pickup coils to build up the sensing array. Such a configuration is depicted in Figure 10. Provided that the Cscan is acquired using two different positions of the sensing array, separated from a distance $d=\sqrt{4 \cdot s_{z}^{2}-s_{z}}$ along $R . d \theta$, the image is acquired with equivalent sampling steps of $s_{z}=0.7 \mathrm{~mm}$ and $s_{\theta}=\sqrt{4 . s_{Z}^{2}-s_{z}} \approx 1.2 \mathrm{~mm}$. This staggered row configuration associated with a two-step acquisition strategy was experimentally evaluated using acquisition parameters $m=66$ and $n=24$, and the sampling and interpolation error was evaluated to be lower than $9 \%$ in the worst imaging case $\left(D_{l}\right.$ at $\left.10 \mathrm{kHz}\right)$, still using $1.4 \mathrm{~mm}$ diameter pickup coils.

\section{Conclusion}

In this study, the experimental feasibility and the first characterization of an EC array probe dedicated to the imaging of millimetric surface breaking defects appearing in bore holes, have been reported. The probe was designed with accurate defect characterization in view. It is constituted of a large inducer generating a uniformly oriented EC flow within the inspected part, and a sensing array used to sense the radial component of the magnetic field resulting at the surface of the wall of the bore hole. An experimental set-up was build in order to validate the imaging performances of such a probe, in a large frequency bandwidth. For the sake of simplicity, a probe prototype featuring a single sensing coil which can be accurately positioned in the sensing area, has been developed in order to evaluate the sensing performances as well as to study the influence of the sensing array configuration. The obtained experimental results proved a good sensing ability of the designed probe in the $10 \mathrm{kHz}-800 \mathrm{kHz}$ frequency range, with a PSNR higher than $36 \mathrm{~dB}$ for a defect featured by a diameter as small as $0.4 \mathrm{~mm}$. Furthermore, a staggered row configuration of the sensing array was proposed so as to significantly reduce (from $35 \%$ to $9 \%$ ) the acquisition error due to the value of the sampling step resulting from the pickup coils geometry. Further works will focus on the implementation of inverse problems algorithms, such as the ones developed and implemented using simulated data in [17], in order to study the performances of the imaging probe in terms of defect characterization performances.

\section{Acknowledgments}

The authors thank MM. Dessendre and Trétout from DassaultAviation for their help.

\section{References}

[1] N. Yusa, H. Huang, K. Miya. Numerical evaluation of the ill-posedness of eddy current problems to size real cracks. NDT and E Int. 40 (2007) 185-191.

[2] D. Prémel, A. Baussard. Eddy current evaluation of 3D flaws in flat conductive materials using a bayesian approach. Inv. Problems. (2002) 1873-89.

[3] J. Pavó, S. Gyimóthy. Adaptative inversion database for electromagnetic nondestructive evaluation. NDT \& E International. 40 (2007) 192-202.

[4] A. Sophian, G. Tian, D. Taylor, J. Rudlin. Design of Pulsed Eddy Current Sensor for Detection of Defects in Aircraft Lap Joints. Sens. Actuators A. 101 (2002) 92-98.

[5] C. Dolabdjian, G. Wache, L. Perez. Improvement in the detection of subsurface fatigue cracks under airframe fasteners using improved rotating giant magneto-resistance magnetometer head. Insight 79(3) (2007) 133-136.

[6] P.-Y. Joubert, Y. Le Diraison. Rotating Field Eddy Current Imaging for the NDE of Buried and Oriented Defects Buried in Riveted Lap-Joints. Sensor Lett. 7(3) (2009) 406-411.

[7] J. Jun, J. Hwang and J. Lee. Quantitative nondestructive evaluation of the Crack on the austenite stainless steel using 
the induced eddy current and the Hall sensor array. in: IMTC 2007 (Ed.) Instrumentation and Measurement Technology Conference - Warsaw, Poland, May 2007.

[8] G. Mook, F. Michel and J. Simonin. Electromagnetic imaging using probe arrays. In: WCNDT 2008 (Ed.). 17th World Conference on Nondestructive Testing, Shanghai, China, October 2008.

[9] B. Marchand, J. M. Decitre, and O. Casula. Flexible and array eddy current probes for fast inspection of complex parts. In: D.O. Thompson and D.E. Chimenti (Eds.), Review of Quantitative Nondestructive Evaluation (29), American Institute of Physics, 2010, pp. 313-320.

[10] A. Yashan, W. Bisle and T. Meier. Inspection of hidden defects in metal-metal joints of aircraft structures using eddy current technique with GMR sensor array. In: ECNDT 2006 (Ed.). European Conference on Nondestructive Testing and Evaluation, Berlin, Germany, 2006.

[11] R. Hamia, S. Cordier, S. Saez, C. Dolabdjian. Giant Magnetosistance magnetometer in Nondestructive Evaluation Eddy current system. Sensor Lett. 7(3) (2009) 437-441.

[12] P. Novotny, P. Sajdl, P. Machac, A magneto optic imager for NDT applications, NDT\&E Int. 37 (2004) 645-649.

[13] P.-Y. Joubert, J. Pinassaud, Linear Magneto-Optic Imager for non-destructive Evaluation. Sens. Actuators A 129 (2006) 126-130.

[14] Hoshikawa, H., Koyama, K., Non-destructive testing of weld zone with a uniform eddy current probe,

Insight: Non-Destructive Testing and Condition Monitoring, Volume 40, Issue 4, 1998, Pages 269-271.
[15] Y. He, F. Luo, and M. Pan, "Defect characterisation based on pulsed eddy current imaging technique," Sensors and Actuators, A: Physical, vol. 164, pp. 1-7, 2010.

[16] V. Thomas, P.-Y. Joubert and E. Vourc'h. Study for the design of an eddy current array probe for the imaging of aeronautical fastener holes. Sensor Lett. 7(3) (2009) 460-465. [17] S. Bausson, V. Thomas, P-Y. Joubert, L. Blanc-Feraud, J. Darbon, G. Aubert, Regularized inversion of a distributed point source model for the reconstruction of defects in eddy currents imaging, COMPEL 30(6) (2011) 1777-1791.

[18] Grandi, G.; Kazimierczuk, M.K.; Massarini, A.; Reggiani, U. Stray capacitances of single-layer air-core inductors for high-frequency applications, In IAS 1996 (Ed.), 31rst Industry Applications Conference. Annual Meeting, vol. 3, March 1996 , pp $1384-1388$.

[19] Y. He, F. Luo, M. Pan, F. Weng, X. Hu, J. Gao, and B. Liu, "Pulsed eddy current technique for defect detection in aircraft riveted structures," NDT and E International, vol. 43, pp. 176-181, 2010.

[20] Metals Handbook, Vol.2 - Properties and Selection: Nonferrous Alloys and Special-Purpose Materials, ASM International 10th Ed. 1990.

[21] Libby HL, Introduction to electromagnetic nondestructive test methods. New York: Robert Kriegger Publisher Company, 1979.

[22] R.P.R. Hasanzadeh, A.R. Moghaddamjoo, S. H. H. Sade Ghi, A.H. Rezaie, and M.Ahmadi, Optimal signal-adaptive maximum likelihood filter for enhancement of defects in eddy current C-scan images NDT and E Int. 41 (2008) 371-377. 